\title{
Successful diagnosis and treatment of biliary cast syndrome after orthotopic liver transplantation by percutaneous transhepatic choledochoscopy, a case report
}

\begin{abstract}
Biliary cast syndrome (BCS) is an unusual complication of orthotopic liver transplantation (OLT). BCS is characterized by multiple intrahepatic biliary strictures, ductal dilatation, intrahepatic abscesses, and biliary anastomotic leakage. The symptoms of BCS usually include high fever, jaundice and cholestatic liver enzyme elevation; similar to the symptoms observed in some patients with intrahepatic bile duct stones. The etiology of cast development is not fully known, and its management is difficult. Limited success using endoscopic retrograde cholangiopancreatography (ERCP) or open exploration to clear casts has been reported, and failure usually results in retransplantation. We describe a diabetic man who developed a BC after deceased donor liver transplantation. Percutaneous transhepatic biliary cholangiography was performed and revealed a filling defect in both the right and left intrahepatic systems, extending down to the anastomosis. Percutaneous transhepatic choledochoscopy proved that it was consistent with BCs, and the casts were removed by stone retrieval basket, foreign body pliers, and adsorber.
\end{abstract}

Volume 2 Issue 4 - 2015

Yu Long Yang, Cheng Zhang, Wu Ping, Hong Wei Zhang, Yue Feng Ma, Mei Ju Lin, Li Jun Shi, Jing Yi Li, Mu Zhao

Department of Biliary Minimally Invasive Surgery, Affiliated

Zhongshan Hospital of Dalian University, China

Correspondence: Yang YL, Department of Biliary Minimally Invasive Surgery, Affiliated Zhongshan Hospital Of Dalian University, Dalian II600 I, Liaoning Province, China, Tel +86-4I I62893656, Fax+86-4I I-62893555, Email YYL516@।26.com

Received: June 29, 2015 | Published: August 24, 2015

\section{Introduction}

A 48-year-old male hepatitis B carrier underwent deceased donor liver transplantation in China for cirrhosis and hepatocellular carcinoma two years ago. His immunosuppressive regimen included corticosteroid and tacrolimus. Immunoglobulin was administered for control of hepatitis B. In view of the persistent liver dysfunction, xanthochromia and skin itching one month ago, endoscopic balloon dilatation was performed and cholangiography showed multiple intrahepatic bile duct strictures. The patient transferred to us for further management of the strictures.

On admission, routine laboratory evaluation showed $67 \mathrm{mmol} / \mathrm{L}$ total bilirubin (reference range , 3-22 mmol/L), $12 \mathrm{mmol} / \mathrm{L}$ conjugated bilirubin (reference range , $0-5 \mathrm{mmol} / \mathrm{L}$ ), 155U/L alanine aminotransferase (reference range , 0-40U/L), 129U/L aspartate aminotransferase (reference range , 0-40U/L), 1038U/L alkaline phosphatase (reference range , 38-126U/L), 884U/L g-glutamyl transferase (reference range , 6-35U/L) and 5.98'109cells/L white blood cell count with $14.6 \mathrm{~s}$ prothrombin time (reference range, 9-14s).

Abdominal ultrasound and computed tomography demonstrated mildly dilated intrahepatic bile duct, normal common bile duct diameter, and normal Doppler flow. Magnetic resonance cholangiopancreatography (MRCP) showed T1 hyperintense and T2 hypointense linear filling defects in the right and left hepatic ducts, extending down to the anastomosis. Cholangiography showed multiple linear filing defects in the proximal hepatic duct proximal to the anastomosis, with extension into the intrahepatic system.

\section{Endoscopic procedure}

In the digital subtraction arteriography suite, with the patient placed in the supine position and under monitored care, percutaneous transhepatic biliary cholangiography was initially performed using a 22-gauge Chiba needle to inject the contrast medium from the right intercostal approach. The puncture site for percutaneous transhepatic biliary drainage was chosen depending on biliary anatomy, position of the $\mathrm{BC}$, and the possibility of puncturing a dilated intrahepatic bile duct under ultrasound. Catheterization of intrahepatic bile ducts was performed in standard fashion. The metal wick-in-needle was removed and a hydrophilic wire was advanced through the metal mandrin into the dilated intrahepatic bile duct. An 8.5-F polyethylene biliary drainage catheter was inserted under the guide of the hydrophilic wire for one week of drainage. Cholangiography revealed a filling defect in both the right and left intrahepatic systems, extending down to the anastomosis, and it was consistent with a BC.

One week after the drainage procedures, the 8.5 -F catheter was replaced with a 10-F catheter, with all side holes in the intrahepatic bile duct. The 12-F, 14-F and 16-F catheters were used in turn once weekly. Nutrition supplementation should be given to avoid protein malnutrition and electrolyte depletion. All drainage procedures were performed with the administration of broad-spectrum antibiotics. The microfiber choledochoscope was passed into the fistulous tract and observed the entire intrahepatic bile duct down to the common bile duct. A stone retrieval basket, foreign body pliers, and adsorber were used to remove the cast until it was not seen under the choledochoscope. Oral $750 \mathrm{mg} / \mathrm{d}$ tauro-ursodesoxycholic acid was used to prevent cholestasis. Follow-up cholangiography revealed no residual filling defects, and the biliary tree was without obvious filling defect. The patient tolerated the procedure well without complications. In the two years after initial removal of the cast, the patient has remained asymptomatic.

\section{Discussion}

BCS has an incidence of $2.5-18 \%$ after OLT. ${ }^{1}$ However, there are limited reports of $\mathrm{BC}$ in the non-transplant situation. ${ }^{2-4}$ Physically and morphologically, a $\mathrm{BC}$ appears as a dark, hardened material in the 
shape of the bile ducts, within the biliary ductal system. Its clinical symptoms usually include high fever, jaundice and cholestatic liver enzyme elevation. Potential consequences include cholangitis and graft damage or loss. Twenty-two percent of the BCS patients require repeat OLT. ${ }^{1}$ However, its mechanism of formation is uncertain. Some of the proposed etiological mechanisms include acute rejection, bile duct damage and ischemia, prolonged cold ischemia time, hemolysis, cholangitis, use of postoperative biliary drainage tubes, biliary infection, fasting, infection, biliary obstruction, supersaturation with cholesterol, mucosal damage, parenteral nutrition, abdominal surgery, head injury/prolonged intensive care, and parenteral nutrition. ${ }^{5-12}$

ERCP, percutaneous transhepatic cholangiography and MRCP are the main diagnostic methods of BCS, and multiple linear filling defects can be seen in the intrahepatic duct system. ${ }^{13-15}$ However, management of BCS is difficult. In the early days, surgical biliary reconstruction was the only available option for treating BCS. ${ }^{16}$ Other treatments such as endoscopic nasobiliary drainage, percutaneous transhepatic drainage, or retransplantation should be considered when complete removal is not feasible or the condition of the recipient deteriorates. Endoscopic cast extraction by ERCP has been described in many reports. However, this method has been usually used in extrahepatic BCS, and management of intrahepatic BCS is difficult, and failure usually results in retransplantation. ${ }^{17,18}$

$\mathrm{BCs}$ can prevent bile drainage, resulting in biliary obstruction and biliary tract infection. Percutaneous transhepatic cholangiography drainage is a common method for drainage of infected bile. After fistula extension, percutaneous transhepatic choledochoscopy can be implemented. We can observe the condition of the intrahepatic and extrahepatic bile ducts and remove multiple BCs with the help of choledochoscope, which is difficult by the conventional methods. ${ }^{19}$ In the process of removing $\mathrm{BCs}$, we found that the $\mathrm{BCs}$ were shaped like the bile ducts, appearing as a hardened, dark material in the biliary ductal system with serious intrahepatic and extrahepatic bile duct injury. Some parts of the biliary mucosa showed patchy or strip defects, and mucosa ulcers were visible sometimes. After completely removing the $\mathrm{BCs}$, the visualization of intrahepatic and extrahepatic bile ducts were improved, and patchy and bile duct mucosa were improved to varying degrees, showing reddish, smooth, rounded lumen and fresh bile secretion, and cholangiography displayed a very clear biliary tree (Figures 1-5).

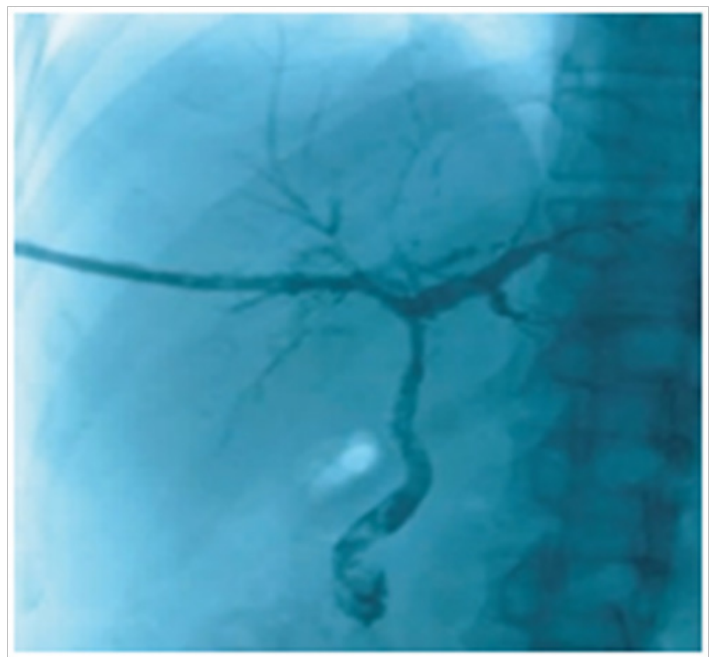

Figure I Cholangiography before BC removal. The intrahepatic bile ducts were poorly visualized, and patchy and tree-like negative shadows were seen.

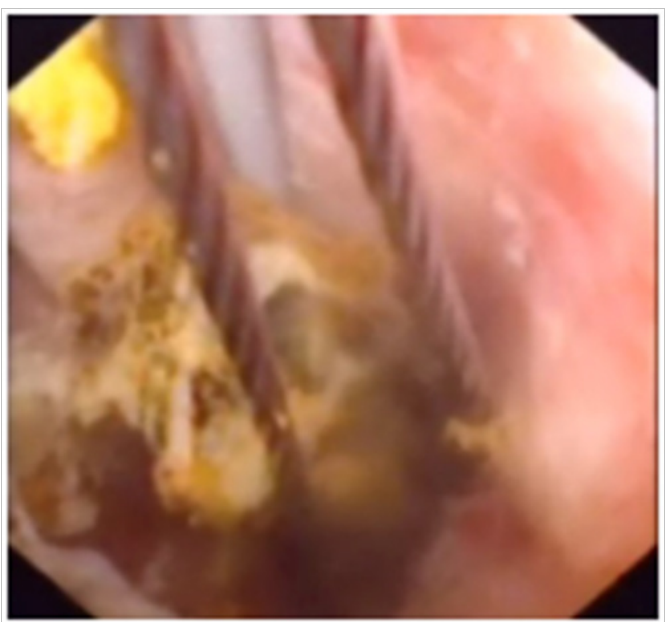

Figure 2 BC removal by choledochoscopy. The figure shows biliary mucosa swelling, erosion and ulceration, and the BC inside was caught by a stone basket.

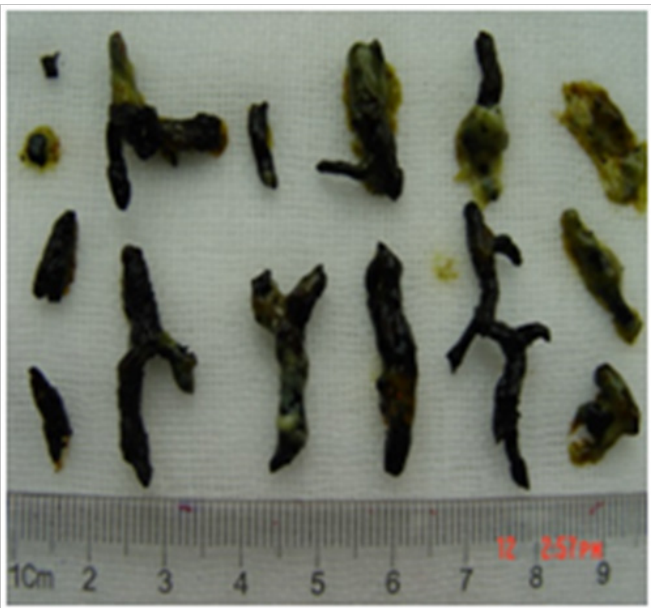

Figure 3 BCs removed by percutaneous transhepatic choledochoscopy. Morphologically, BCs are shaped like bile ducts, appearing as hardened, dark material in the biliary ductal system.

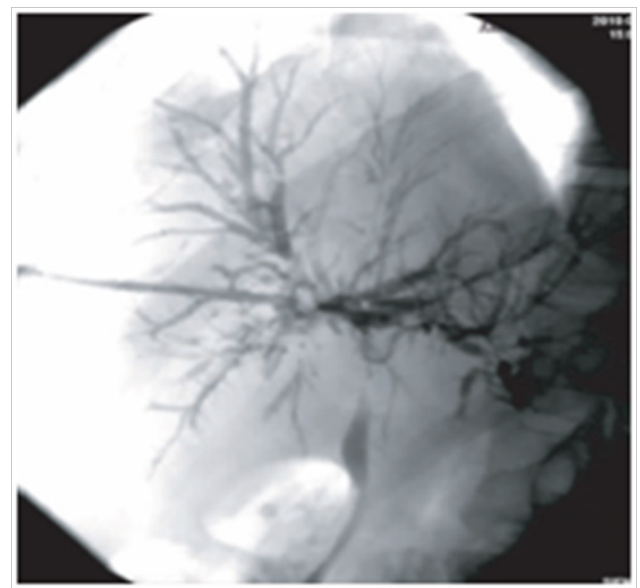

Figure 4 Cholangiography after BC removal. The first cholangiographic findings of the same patient after endoscopic therapy. Visualization of intrahepatic and extrahepatic bile ducts was improved, and patchy and treelike negative shadows partially vanished. 


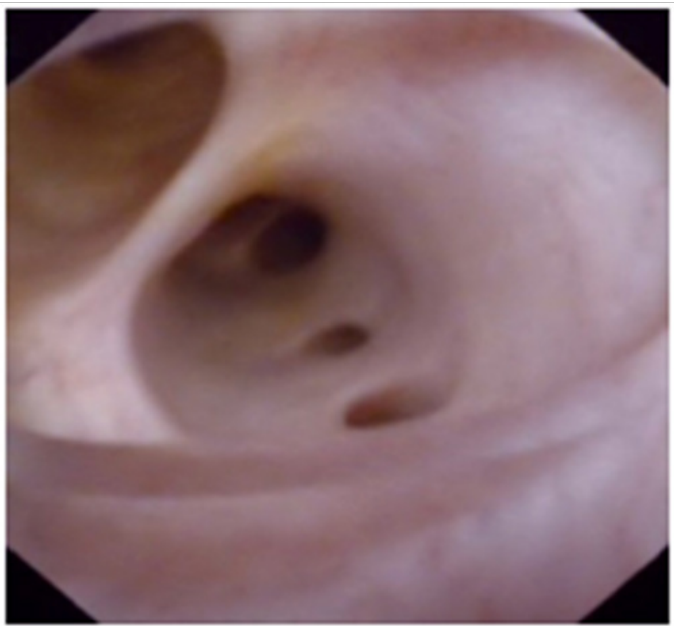

Figure 5 Choledochoscopic investigation after BC removal. The figure shows reddish, smooth, rounded lumen and fresh bile secretion.

There are many considerations for percutaneous transhepatic choledochoscopy. For different location of the $\mathrm{BC}$, the appropriate puncture site should be selected. If the BC is in the right hepatic duct, we should puncture the left bile duct. This can reduce the difficulty of choledochoscopy and avoid the angle between the biliary duct and the choledochoscope. Choledochoscopic techniques should be used to reduce the incidence of bile duct mucosal damage and bleeding. BCs are often combined with bile duct stricture, located at the proximal end of the cast. Before removing the $\mathrm{BC}$, bile duct stricture should be resolved. ${ }^{19}$ Selective endoscopic biliary visualization can be used to find occult BCS, and balloon dilatation or endoscopic high frequency electric cutting can resolve the biliary duct stricture. ${ }^{15,20}$

Percutaneous transhepatic choledochoscopy not only has a diagnostic role, but also can achieve the desired therapeutic effect with minimal invasion, repetition, and patient tolerance. With the popularity of endoscopic techniques and their improved operation by clinical physicians, an increasing number of patients with biliary complications after liver transplantation will benefit from this technique.

\section{Acknowledgments}

None.

\section{Conflicts of interest}

Author declares there are no conflicts of interest

\section{Funding}

None.

\section{References}

1. Gor NV, Levy RM, Ahn J, et al. Biliary Cast Syndrome Following Liver Transplantation: Predictive Factors and Clinical Outcomes. Liver Transpl. 2008;14(10):1466-1472.

2. Dziurkowska-Marek A, Hartleb M, Marek TA, et al. Fatal case of biliary cast syndrome in nontransplant patient. Endoscopy. 2009;41 (Suppl 2):E256.
3. Saleem A, Baron TH. Successful endoscopic treatment of biliary cast syndrome in an orthotopic liver transplant patient with a Roux-en-Y anastomosis via balloon enteroscopy. Liver Transpl. 2010;16(4):527529.

4. Jain PA, Gandhi VV, Desai P, et al. Recurrent cholangitis in the tropics worm or cast? J Postgrad Med. 2010;56(4):287-289.

5. Waldram R, Williams R, Calne RY. Bile composition and bile cast formation after transplantation of the liver in man. Transplantation. 1975;19(5):382-387.

6. McMaster P, Herbertson BM, Cusick C, et al. The development of biliary "sludge" following liver transplantation. Transplant Proc. 1979;11(1):262-266.

7. McMaster P, Walton RM, White DG, et al. The influence of ischaemia on the biliary tract. Br J Surg. 1980;67(5):321-324.

8. Parry SD, Muiesan P. Cholangiopathy and the biliary cast syndrome Eur J Gastroenterol Hepatol. 2003;15(4):341-343.

9. Byrne MF, Chong HI, O'Donovan D, et al. Idiopathic cholangiopathy in a biliary cast syndrome necessitating liver transplantation following head trauma. Eur J Gastroenterol Hepatol. 2003;15(4):415-417.

10. Shah JN, Haigh WG, Lee SP, et al. Biliary casts after orthotopic liver transplantation: clinical factors, treatment, biochemical analysis. Am $J$ Gastroenterol. 2003;98(8):1861-1867.

11. Zimmer V, Raedle J, Treiber G, et al. Biliary cast syndrome in sclerosing cholangitis. Dig Liver Dis. 2011;43(2):e4.

12. Yang YL, Shi LJ, Lin MJ, et al. Clinical analysis and significance of cholangiography for biliary cast/stone after orthotopic liver transplantation. J Nanosci Nanotechnolo. 2013;13(1):171-177.

13. Bak SH, Choi HS, Yang SY, et al. Obstructive jaundice due to biliary cast syndrome followed by orthotopic liver transplantation. Korean J Gastroentero. 2006;48(2):119-123.

14. Navaneethan U, Venkatesh PG, Al Mohajer M, et al. Successful diagnosis and management of biliary cast syndrome in a liver transplant patient using single operator cholangioscopy JOP. 2011;12(5):461-463.

15. Yang YL, Zhang C, Lin MJ, et al. Biliary casts after liver transplantation: morphology and biochemical analysis. World $J$ Gastroenterol. 2013;19(43):7772-7777.

16. Sheng R, Ramirez CB, Zajko AB, et al. Biliary stones and sludge in liver transplant patients: a 13-year experience. Radiology. 1996;198(1):243247.

17. Kusnierz K, Nowakowska-Dulawa E, et al. Biliary cast syndrome in a non-transplant patient with acute pancreatitis. Dig Liver Dis. 2012;44(6):534-535.

18. Lopez-Benitez R, Wielputz MO, Bryant MG, et al. Percutaneous treatment of biliary cast syndrome after orthotopic liver transplantation: comparison of mechanical versus hydraulic rheolytic cast extraction. Cardiovasc Intervent Radiol. 2011;34(5):998-1005.

19. Yang Yu-long, Liu Zhen-wen. Analysis on biliary complications after orthotopic liver transplantation using biliary tract endoscopy. Journal of Clinical Rehabilitative Tissue Engineering Research 2010;14(18):3417-3420.

20. Yang Yu-long, Chen Hai-long, Tan Wen-xiang, et al. Balloon dilatation plus support tube for treatment of biliary stricture after orthotopic liver transplantation by using the endoscope technique. Journal of Clinical Rehabilitative Tissue Engineering Research. 2008;12(31):6181-6186. 